\title{
A review of the genus Zubovskya Dovnar-Zapolskij, 1932 (Orthoptera: Catantopidae, Conophyminae)
}

\author{
Xinjiang Li, Baoping Li \& Xiangchu Yin
}

\begin{abstract}
Li, X. J., Li, B. P. \& Yin, X. C. 2015: A review of the genus Zubovskya DovnarZapolskij, 1932 (Orthoptera: Catantopidae, Conophyminae). — Entomol. Fennica 26: $110-116$.
\end{abstract}

The genus Zubovskya Dovnar-Zapolskij, 1932 is reviewed. This genus consists of six species. Two new species are described from China: Zubovskya xiai $\mathbf{s p .} \mathbf{n}$. from Heilongjiang Province and Zubovskya eyouqiensis sp. n. from Inner Mongolia Autonomous Region. Two species are transferred from Zubovskya to the genera Bienkoa Mishchenko, 1950 and Kingdonella Uvarov, 1933. Therefore, two new combinations are proposed: Podisma morii Bey-Bienko, $1931=$ Bienkoa morii (Bey-Bienko, 1931), comb. n.; Zubovskya mongolica Storozhenko, 1986 = Kingdonella mongolica (Storozhenko, 1986), comb. n. Key to the species of Zubovskya is given.

X. J. Li \& X. C. Yin (*corresponding author), The Key Laboratory of Zoological Systematics and Application, College of Life Sciences, Hebei University, Baoding, Hebei 071002, China; and Northwest Plateau Institute of Biology, Chinese Academy of Sciences, Xining, 810001, China; * Corresponding author's e-mail: yxch@hbu.edu.cn

B. P. Li, Department of Entomology, Nanjing Agricultural University, Nanjing, 210095, China

Received 22 January 2015, accepted 17 February 2015

\section{Introduction}

The genus Zubovskya was established by Dovnar-Zapolskij (1932) in the subfamily Catantopinae and it was traditionally considered belonging to the tribe Podismini (Bey-Bienko \& Mishchenko 1951, Storozhenko 1986, Huang 1987, Yin et al. 1996, Storozhenko 1998, Li et al. 2006). Recently the genus was transferred to the tribe Plotnikovini (subfamily Conophyminae) and divided in two genera, Zubovskya and Eozubovskya Li et Yin, 2009 (Li \& Yin 2009). As the result, the genus Zubovskya consists currently of six species distributed mainly in Korea, Mongolia, Russian Far East and China (Heilongjiang,
Jilin, Liaoning, and Inner Mongolia Autonomous Region). In the present paper we describe two new species from China, as well as two species are transferred from Zubovskya to other genera of Conophyminae.

\section{Material and methods}

The specimens were collected mainly from China and deposited in Northwest Plateau Institute of Biology, Chinese Academy of Sciences and College of Life Sciences, Hebei University. For the other species that are distributed in Korea, Mongolia, and Russian Far East, we checked the pho- 
tos of the type specimens on the website (Eades \& Otte 2014) and the original papers describing them (Zubovski 1900, Ikonnikov 1911, BeyBienko 1931, Storozhenko 1986, Huang 1987). The type specimens are deposited in Northwest Plateau Institute of Biology, Chinese Academy of Sciences, Xining.

Descriptions and measurements were made under a stereomicroscope (Leica M 205 A, LAS V 4.0). All photos were taken using a SRL camera (Canon 5D Mark II) and developed with Adobe Photoshop version 9.0 software.

\section{Taxonomy}

\subsection{Genus Zubovskya Dovnar-Zapolskij, 1932}

Zubovskya Dovnar-Zapolskij, 1932: 255, 258. Zubovskia Bey-Bienko \& Mishchenko, 1951: 139.

Type species. Podisma parvula Ikonnikov, 1911, by original designation. = Zubovskya parvula (Ikonnikov, 1911).

Diagnosis. Body small to middle sized; head shorter than length of pronotum; anterior margin of fastigium intact in dorsal view, without emargination; antennae filiform, in both sexes long, extending far beyond poster margin of pronotum, reaching or not base of hind femora; pronotum cylindrical, posterior margin of pronotum intact or slightly concaved; lateral carinae absent, median carina low, slightly cut by three transverse sulci; prozona about 2.0-3.5 times longer than metazona; prosternal process conical, apex pointed or slightly pointed; lateral lobes of mesosternum wider than long, mesosternal interspace squarish; metasternal lobes widely separated; tegmina and wings absent; upper keel of hind femur smooth, kneelobes rounded; external apical spine of hind tibia absent; tympanum absent on first abdominal tergite; tenth tergite with distinct furculae and epiproct triangular with rounded apex in male; cerci of male slightly s-shaped in dorsal view, widened at base and apex part in lateral view; ovipositor with 2 teeth on tip of valves.

Species composition. The genus consists of four species, namely Zubovskya parvula (Ikonnikov, 1911), Z. dolichocercata Huang, 1987, Z. koeppeni (Zubovsky, 1900), and Z. brachycer- cata Huang, 1987 (Eades \& Otte 2014). The latter species was considered as synonym of $Z$. parvula (Storozhenko, 1998), but is a valid species (Li et al. 2006). In addition, two new species are described below.

Notes. Based on having an external apical spine on hind tibia, first abdominal tergite with tympanum, and pronotum with longitudinal carina Zubovskya morii (Bey-Bienko, 1931) is transferred here to the genus Bienkoa Mishchenko, 1950. Based on absences of external apical spine on hind tibia, well defined lateral carinae on pronotum and lack of tympanum, $Z u$ bovskya mongolica Storozhenko, 1986 is moved to Kingdonella Uvarov, 1933. Therefore two new combinations are proposed here: Podisma morii Bey-Bienko, 1931 = Bienkoa morii (Bey-Bienko, 1931), comb. n.; Zubovskya mongolica Storozhenko, 1986 = Kingdonella mongolica (Storozhenko, 1986), comb. n.

\subsection{List of species with description of new taxa}

Zubovskya koepenni (Zubowsky, 1900)

Podisma koeppeni Zubowsky, 1900: 20 (lectotype male, Russia: Transbaikalia, Tylnoi River; deposited in Zoological Institute, Russian Academy of Sciences, St. Petersburg (ZIN)).

Zubovskya koeppeni: Dovnar-Zapolskij, 1932: 265, 261, 267.

Distribution. Russia (Siberia from Altai Mountains to Transbaikalia).

Zubovskya parvula (Ikonnikov, 1911)

Podisma parvula Ikonnikov, 1911: 260 (neotype male, Russia: Primorye, 20 km E SpasskDalny; deposited in ZIN).

Zubovskya parvula: Dovnar-Zapolskij, 1932: 256, 261, 267.

Distribution. Russia (Far East), China (Heilongjiang, Jilin).

Zubovskya brachycercata (Huang, 1987)

Zubovskia brachycercata Huang, 1987: 309 (holotype male, China: Heilongjiang, Jingpohu; deposited in Institute of Zoology, Chinese Academy of Sciences, Beijing (IZCAS)).

Distribution. China (Heilongjiang). 


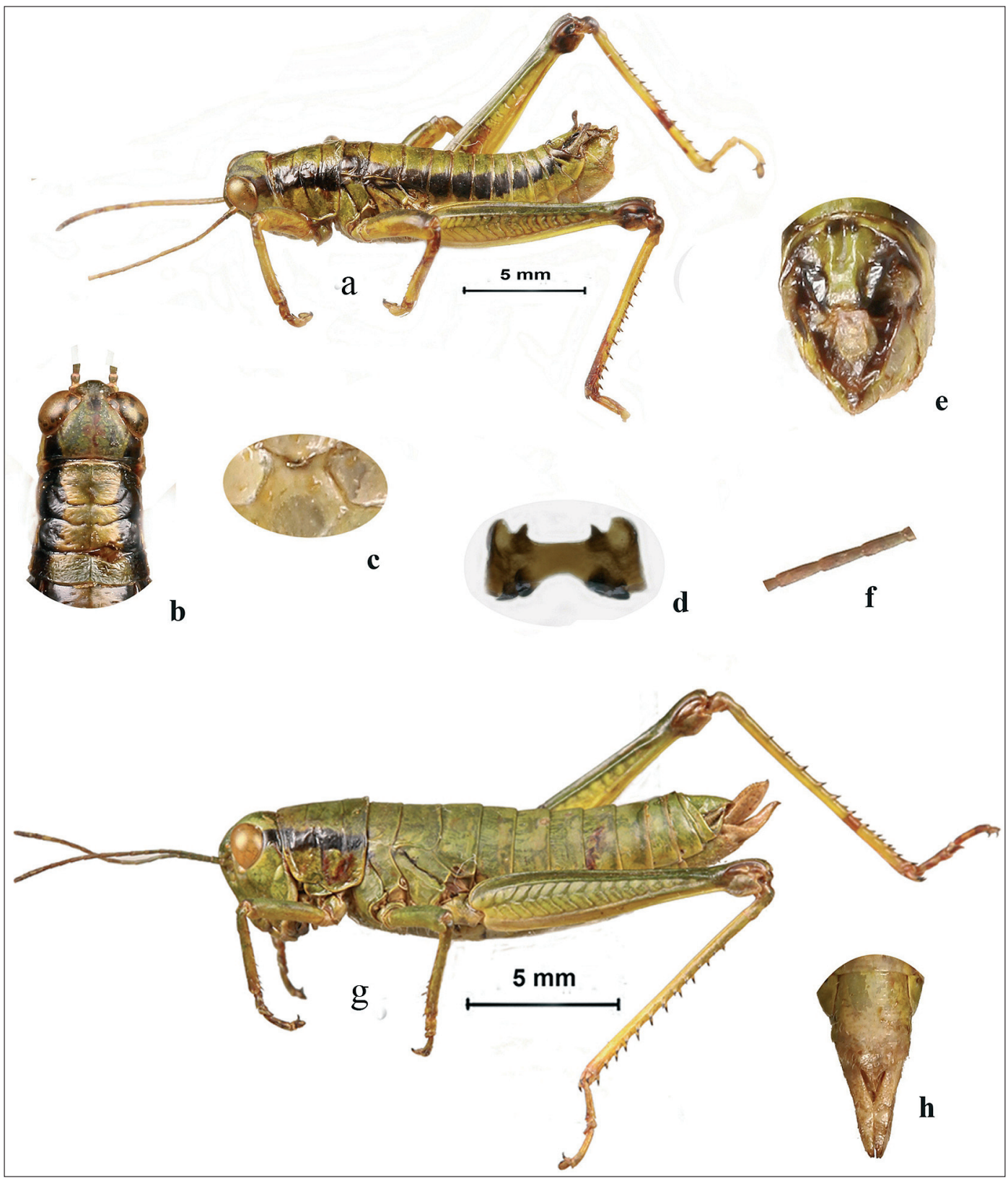

Fig. 1. Photo of Zubovskya xiai sp. n. - a. Male body in lateral view. - b. Male head and pronotum in dorsal view. - c. Male mesosternum in ventral view. - d. Epiphallus. - e. End of male abdomen in dorsal view. - f. Female antenna, middle part. - g. Female body in dorsal view. $-\mathrm{h}$. End of female abdomen in ventral view.

Zubovskya dolichocercata (Huang, 1987)

Zubovskia dolichocercata Huang, 1987: 308 (holotype male, China: Heilongjiang, Wuchang; deposited in IZCAS).

Distribution. China (Heilongjiang).
Zubovskya xiai sp. n. (Fig. 1, Table 1)

Type material. Holotype male: China, Dailing, Heilongjiang province; 47 12 '18' N, 12854'34” E, $800 \mathrm{~m}$ a.s.1., 21.VIII.1984, XiangChu Yin and Bao-Ping Li leg. Paratypes, one 
Table 1. Comparison between Zubovskya xiai sp. n. and Z. parvula.

\begin{tabular}{|c|c|c|}
\hline Characters & Z. xiai sp. n. & Z. parvula \\
\hline Length of prozona / length of metazona & 3.0 & $2.6-2.7$ \\
\hline Posterior margin of epiproct in male & Almost straight & Projecting in the middle \\
\hline Distance between two furculae & Twice of width of a furcular & Narrower than width of a furcula \\
\hline Length / width of hind femur & 4.6 & $5.0-5.4$ \\
\hline
\end{tabular}

male and two females: the same data as for holotype. Holotype and paratypes deposited in Northwest Plateau Institute of Biology, Chinese Academy of Sciences (NPIBCAS), Xining.

Diagnosis. The new species is similar to $Z$. parvula (Ikoninikov, 1911). The major differences are listed in Table 1.

Description. Male: Body medium in size (Fig. 1a). Head shorter than pronotum (Fig. 1b), vertex shorter, apex wider. Face slightly oblique in profile. Antennae filiform, with 22 segments, its longest medial segment 3.3 times as long as wide (Fig. 1f). Eyes ellipse, vertical diameter of eyes 1.4 times horizontal diameter of eyes and 1.5 times subocular furrow. Pronotum cylindrical, anterior margin straight, hind margin almost straight; median and lateral carina indistinct, pronotum cut by three transverse sulci, prozona 3.0 times longer than metazona. Prosternal process conical, apex rounded. Mesosternal interspace wide, 1.3 times wider than long (Fig. 1c) and lateral lobes of metasternum separated. Wings absent. Upper keel of hind femur smooth, its length 4.6 times of its maximum width, end of lower knee lobes rounded. Hind tibia with 11 spines on inner side and 10 spines on outer sides, external apical spine absent. First tarsus segment about three times longer than second one. Tympanum absent. Basal part of epiproct with longitudinal groove in middle, furculae small, separated, distance between two furculae twice as width of a furcula. Cercus conical with rounded apex, extending over tip of epiproct. Subgenital plate short, apex conical and acute (Fig. 1e). Epiphallus shown in Fig. 1d.

Female: Body (Fig. 1g) more robust than in male. Vertical diameter of eyes 1.4 times longer than horizontal diameter and 1.2 times longer than subocular furrow. Prozona 2.7 times longer than metazoan. Cercus short and conical, not reaching end of epiproct. Length of subgenital plate longer than width, posterior apical part widened, hind margin acute in middle (Fig. 1h). Ovipositor valve widened in middle, apex acute, upper valve with teeth on upper margin.

Coloration: General appearance yellowish green. Antennae yellowish brown. Eyes brown. Black postocular band present in both sexes. Hind femur yellowish green, inner knee lobe black. Hind tibia yellow, basally black. Abdomen yellowish green. Male subgenital plate yellow.

Measurements (in $\mathrm{mm}$ ): Body length: male 16.2-16.5, female 20.3-21.1. Length of pronotum: male 3.8-4.2, female 4.3-4.5. Length of hind femur: male 9.0-10.4, female 11.2-12.8.

Etymology. The species is named in honor of Prof. Kailing Xia (1916-2013) on his centenary birth anniversary. He was our career mentor and an eminent insect taxonomist.

Zubovskya eyouqiensis sp. n. (Fig. 2, Table 2) Type material. Holotype male: China, Inner Mongolia Autonomous Region, Eyouqi, $50^{\circ} 23^{\prime} 24 " \mathrm{~N}, 120^{\circ} 18^{\prime} 43$ ' E, $700 \mathrm{~m}$ a.s.1., 14.VIII.1984, Xiang-Chu Yin and Bao-Ping Li leg. Paratypes, one male and two females: the same data as for holotype. Holotype and paratypes deposited in Northwest Plateau Institute of Biology, Chinese Academy of Sciences (NPIBCAS), Xining.

Diagnosis. The new species is similar to $Z$. koepenni (Zubowsky, 1900). The major differences are listed in Table 2.

Description. Male: Body medium sized (Fig. 2b). Head shorter than pronotum, vertex shorter (Fig. 2d), apex straight. Face distinctly oblique in profile. Antennae filiform with 20 segments, its longest medial segment 2.2 times as long as wide (Fig. 2a). Eyes ellipse, vertical diameter 1.3 times longer than horizontal diameter and 1.4 times subocular groove. Pronotum cylindrical, anterior margin excised slightly in middle (Fig. 2d), hind 


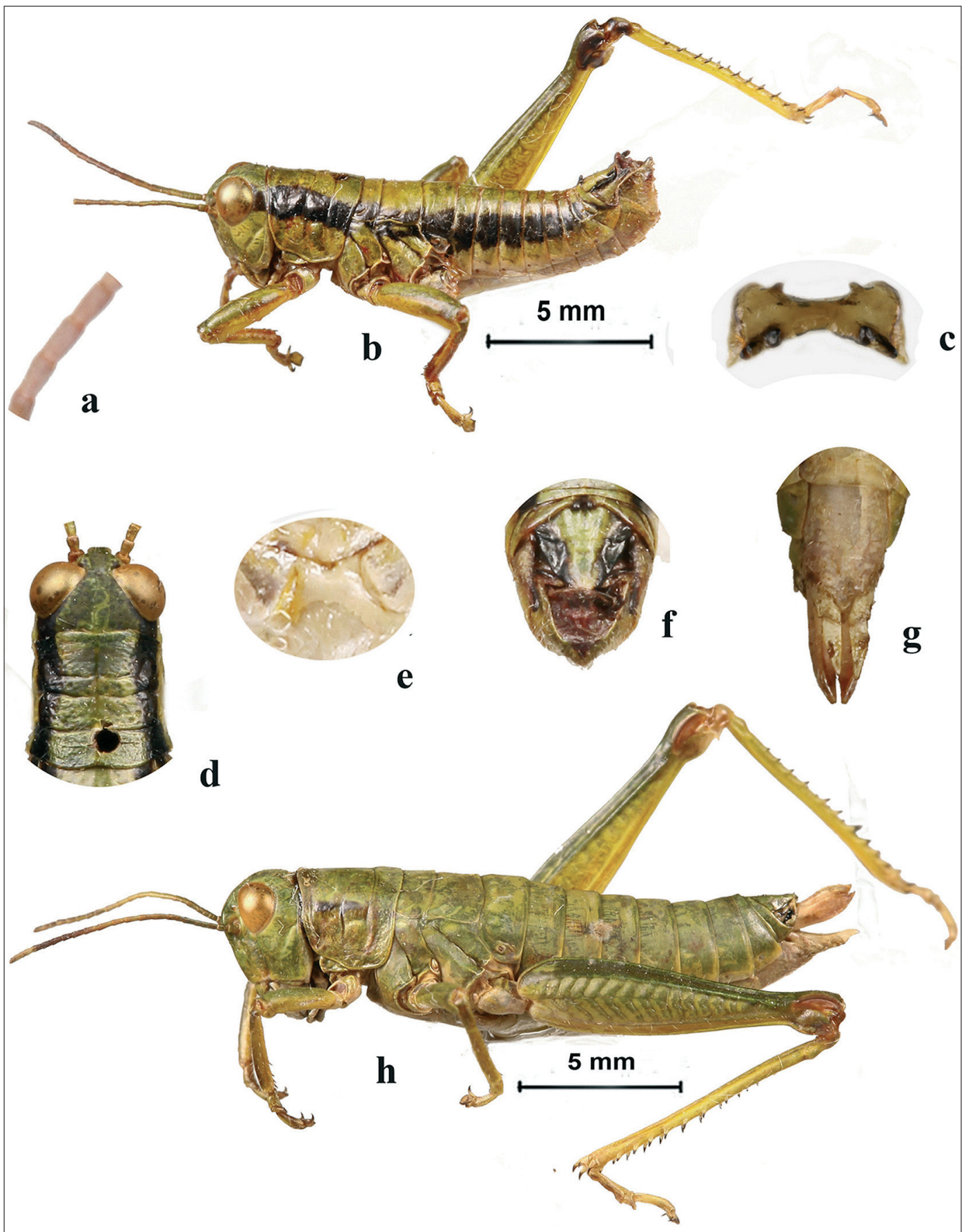

Fig. 2. Photo of Zubovskya eyouqiensis sp. n. - a. Male antenna, middle part. - b. Male body in lateral view. - c. Epiphallus. - d. Male head and pronotum in dorsal view. - e. Male mesosternum in ventral view. $-\mathrm{f}$. End of male abdomen in dorsal view. g. - End of female abdomen in ventral view. - h. Female body in lateral view.

margin concave in middle; median and lateral carina indistinct, pronotum cut by three transverse sulci, length of prozona 2.6 times length of metazona. Prosternal process conical, apex rounded. Mezosternal interspace wide 1.3 times wider than long (Fig. 2e), lateral lobes of meta- 
Table 2. Comparison between Zubovskya eyouqiensis sp. $\mathbf{n}$. and Z. koepenni.

\begin{tabular}{lll}
\hline Characters & Z. eyouqiensis sp. $\mathbf{n}$. & Z. koepenni \\
\hline Length of prozona / length of metazona & $2.6-3.0$ & $2.3-2.5$ \\
Length / max width of hind femur & 4.6 & $4.8-5.4$ \\
Longitudinal groove of male epiproct & Long in middle, & Short in middle, \\
& overall widened & overall narrower \\
Length of furculae in relation to epiproct & $1 / 12$ & $1 / 6$ \\
\hline
\end{tabular}

sternum separated. Wings absent. Upper keel of hind femur smooth, its length 4.3 times of its maximum width, end of lower knee lobes rounded. Hind tibia with 10 spines on inner and 9 spines on outer sides, external apical spine absent. First tarsus segment about three times longer than second. Tympanum absent. Epiproct with a longitudinal groove in middle, overall widened, near middle on both sides with wide projections. Furculae small, separated, distance between two furculae equal to width of a furcula. Cercus conical (Fig. 2f), base distinctly wide, extending over tip of epiproct. Subgenital plate short and conical, apex obtuse. Epiphallus as in Fig. 2c.

Female: Body more robust (Fig. 2h). Vertical diameter of eyes 1.3 times horizontal diameter and 1.1 times subocular groove. Length of prozona 3.0 times length of metazona. Cercus short and conical, not reaching tip of epiproct. Subgenital plate longer than wide, hind margin with an acute projection in middle (Fig. 2g). Ovipositor valve slender, apex acute, outer margin almost smooth.

Coloration: In general appearance body green in male. Head green. Eyes brown. Antennae yellowish brown. Postocular black band present, extending to abdomen. Outer of hind femur green in middle area and dark black green in upper area, inner knee lobe black. Hind tibia yellow, base black. Abdomen green. Subgenital plate yellowish brown. Female without postocular black band, otherwise as male.

Measurements (in mm): Length of body: male 17.1, female 26.1. Length of pronotum: male 3.5, female 5.1. Length of hind femur: male 10.3, female 13.6.

Etymology. The species is named for the type locality, Eyouqi, Inner Mongolia Autonomous Region.

\subsection{Key to species of Zubovskya}

1. Furculae in male very large, about equal to $1 / 4$ length or slightly less than $1 / 4$ of epiproct 2

- Furculae in male smaller, about equal to $1 / 6$ $1 / 5$ of epiproct 3

2. Antennae slender, reaching base of hind femur, length of a segment in middle of antennae about 3.1-3.8 times width. Hind femur relatively slender, length about 5.3-6.1 times width. Cerci of male narrow and long, length 2.8-3.4 times maximum width

Z. dolichocercata

- Antennae not reaching base of hind femur, the length of a segment in middle of antennae about 4.3-5.1 times width. Cerci of male stout and short, length 2.1-2.7 times maximum width Z. brachycercata

3. Antennae slender, reaching base of hind femur, length of a segment in middle of antennae about 2.5-3.3 times width 4

- Antennae relatively stout and short, far from reaching base of hind femur, length of a segment in middle of antennae about 2.0-2.5 times width

4. Hind femur slender, length about 5.5-6.2 times width. Prozona of pronotum 2.6-2.7 times metazona in length. Posterior margin of epiproct in male projected in the middle, distance between two furculae narrower than width of a furcula in male Z. parvula

- Hind femur slender, length about 4.6 times width. Prozona of pronotum 3.0 times metazona in length. Posterior margin of epiproct in male almost straight, distance between two furculae 2 times width of a furcula in male

Z. xiai sp.n.

5. Prozona of pronotum longer, its length about 2.5-3.0 times metazona. Length of hind fe- 
mur 4.2-4.6 times width. Epiproct with a long longitudinal groove in middle, overall widened. Furculae equal $1 / 12$ of epiproct in length

Z. eyouqiensis $\mathbf{s p .} \mathbf{n}$.

- Prozona of ponotum longer, its length about 2.3-2.5 times metazona. Hind femur slender, its length 4.8-5.4 times width. Epiproct with short longitudinal groove in middle, narrower. Furculae equal 1/6 of epiproct in length

Z. koepenni

Acknowledgements. This study is funded by the National Natural Science Foundation of China (No. 31372153), Natural Foundation of Hebei Province (No. C2013201148) and Run Subsidy of Hebei Province's Key Laboratory(No. 14967611D).

\section{References}

Bey-Bienko, G. J. 1931: On some Orthoptera from Northern Korea. - Boletín de la Real Sociedad Española de Historia Natural 31: 673-678.

Bey-Bienko, G. J. \& Mishchenko, L. L. 1951: Locusts and grasshoppers of the USSR and adjacent countries. Vol. 1. - Nauka Publishing House, Moscow-Leningrad, 380 p. [In Russian.]

Eades, D. C. \& Otte, D. 2014: Orthoptera Species File Online. Version 5.0/5.0. [www document]. URL http:// Orthoptera.Species File.org. (Site visited on 12 December 2014)

Dovnar-Zapolskij, D. P. 1932: Zur Kenntnis der palearktischen Podismini (Orthoptera, Acridoidea). - Trudy Zoologitscheskogo Instituta, Akademiia Nauk SSSR, Leningrad [= Proceedings of the Zoological Institute, USSR Academy of Sciences, Leningrad] 1(3-4): 253268. [In Russian with German summary.]
Huang, C. M. 1987: Three new species of the genus $Z u$ bovskia Dovnar-Zapolskij. 1932 from China (Acrididae; Catantopinae). — Acta Entomologica Sinica 30(3): 307-312.

Ikonnikov, N. 1911: Zur kenntnis der Acridiodeen Sibiriens. - Annuaire du Musée Zoologique de l'Académie Impériale des Sciences de St.-Pétersbourg 16: 242-270.

Li, H. C., Xia K. L., Bi D. Y., Jin, X. B., Huang, C. M., Yin, X. C., Zheng, Z. M., Lian, Z. M., You, Q. J., Zhang, F. L. \& Li, T. S. 2006: Orthoptera. Acridoidea. Catantopidae. - Fauna Sinica. Insecta. Vol. 43. Science Press, Beijing, 736 p. [In Chinese with English summary.]

Li, X. J. \& Yin, X. C. 2009: A taxonomic study of the subfamily Conophyminae (Orthoptera: Caelifera: Acridoidea) from Eurasia. - Acta Entomologica Sinica 52(10): 1139-1145.

Mishchenko, L. L. 1952. Fauna of USSR. Orthoptera. IV. 2. Locusts and grasshoppers (Catantopinae). - USSR Academy of Sciences, Leningrad. 610 p. [In Russian.]

Yin, X. C., Shi, J. P. \& Yin, Z. 1996: Synonymic Catalogue of Grasshoppers and their Allies of the World (Orthoptera: Caelifera). - China Forestry Publishing House, Beijing, $1266 \mathrm{p}$.

Storozhenko, S. Yu. 1986: Revision of the genus $Z u$ bovskaya Dov.-Zap. (Orthoptera: Acrididae). - Trudy Zoologicheskogo Instituta, Akademiia Nauk SSSR, Leningrad [ = Proceedings of the Zoological Institute, USSR Academy of Sciences, Leningrad] 143: 47-58. [In Russian.]

Storozhenko, S. Yu. 1998: New data on grasshoppers (Orthoptera: Acrididae) of North-East Asia. - Far Eastern Entomologist 64: 6-8.

Zubowsky, N. 1900: Beitrag zur Kenntniss der sibirischen Acridiodeen. - Trudy Russkogo Entomologicheskogo Obshchestva [= Horae Societatis Entomologicae Rossicae] 34(1-2): 1-23. 